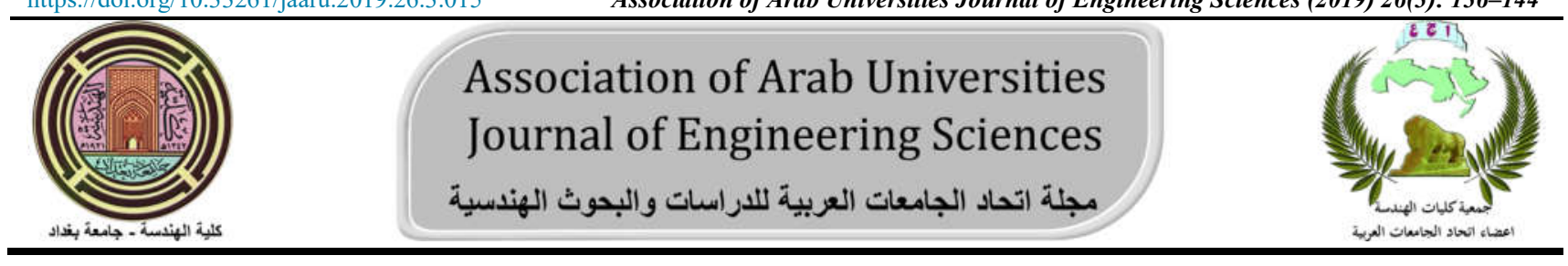

اتقييم المحتوى الحضري بيئياً بالاعتمـاد على الوسط الرقمي

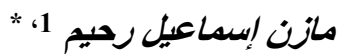

قسم هندسة العمارة، جامعة بغداد، بغداد، العراق، archi.mazin@yahoo.com

"الباحث المثثل: مازن إسماعيل رحيم، البريب الالكترونسي: archi.mazin@yahoo.com

شر في: 31 ت آب 2019

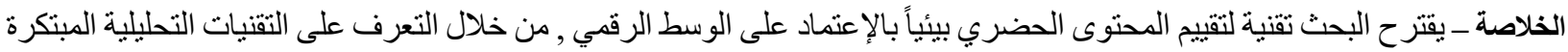

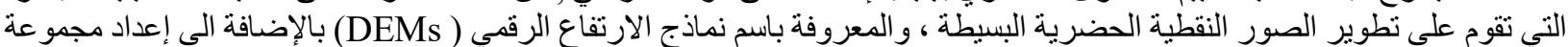

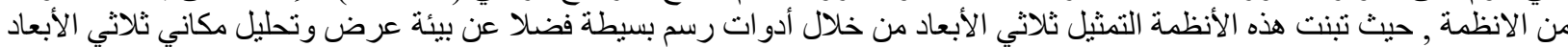

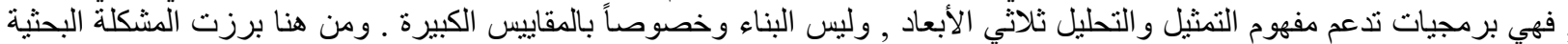

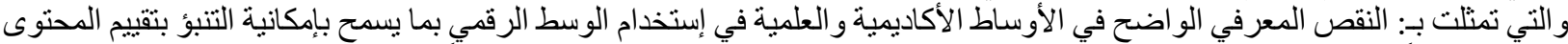

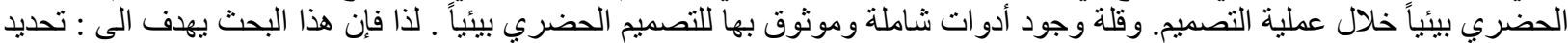

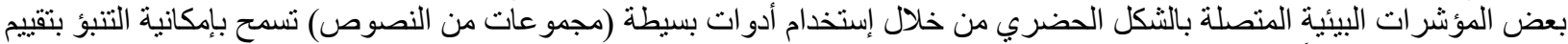

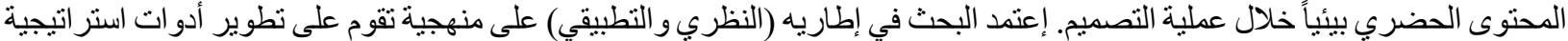
فعالة لتحليل وتخطيط الثكل الحضري ، وقياس المؤشر ات البيئية وصو لاً الى الإستنتاجات و التوصيات.

\section{4}

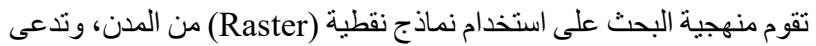

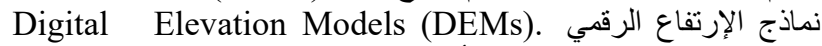

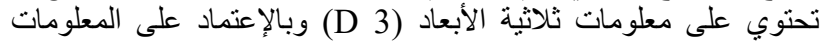

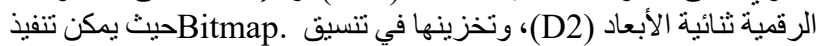

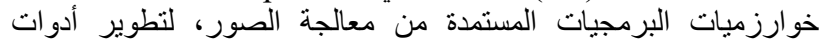

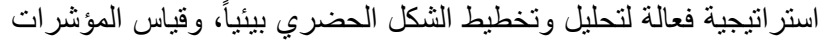

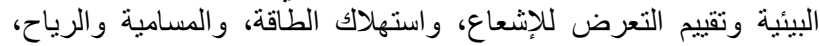

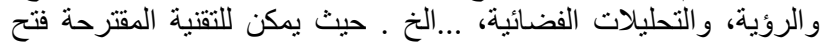

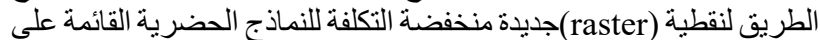

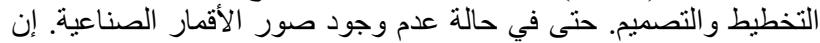

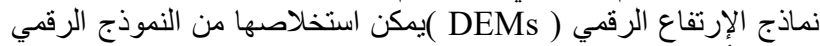

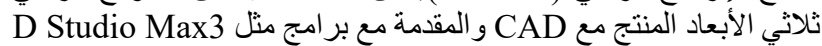

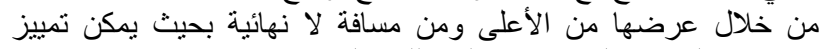

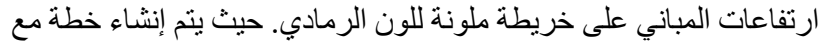

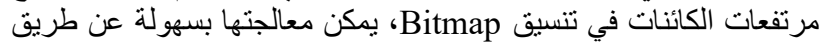

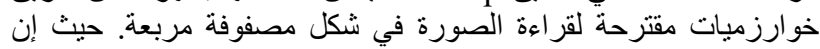

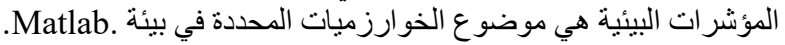

$$
\text { التصديم الحضري: }
$$

يعرف التصميم الحضري على إنه: فن تصميم السياقية [4] ويعرف أيضاً

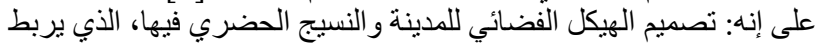

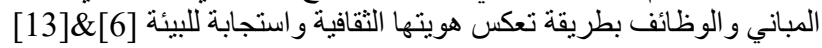

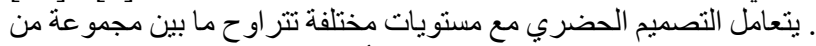

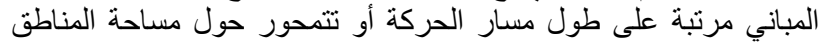

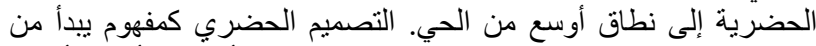

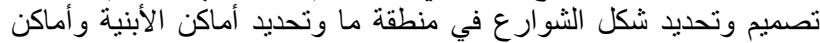

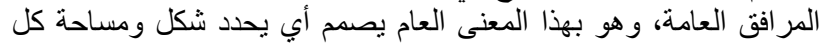

[4] في المر في المدينة.

\section{1. \\ 1.1}

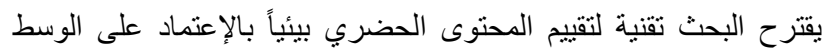

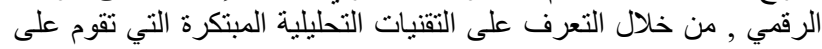

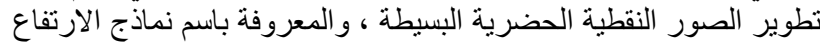

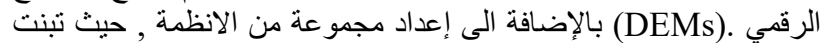

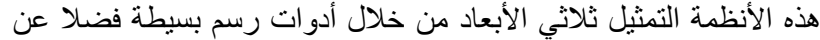

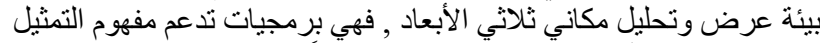

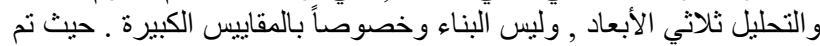

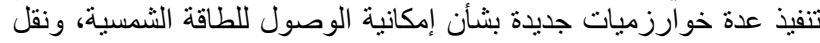

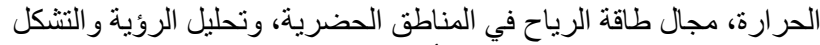

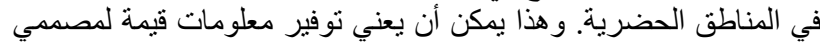
ومخططي المدن ، وتحسنا كبيرا في مجال كفاءة استخدام الطاقة و والراحةً البيئية . وملية

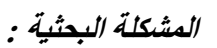

1.2

تمثلت بالنقص المعرفي الواضح في الأوساط الأكاديمية و العلمية في إستخدام

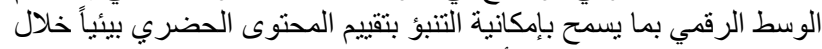

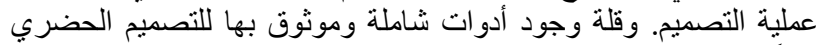

$$
\text { } 1.3
$$

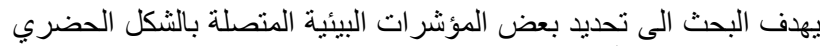

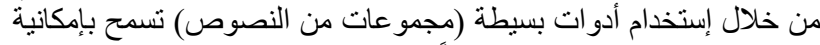
التنبؤ بتقييم المحتوى الحضري بيئياً خلال عملية التصميج. 
جدول بإستخدام برنامج Excel لجمع كل النتائج الناشئة من الحسابات .

$$
2.2
$$

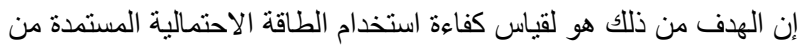

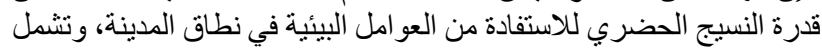

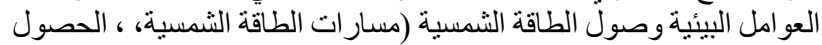

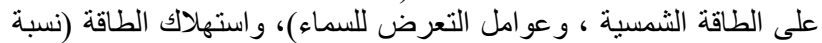
السطح إلى الحجم والمناطق السلبية / و المناطق غير السئة السلبية)، التهوية،

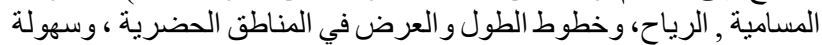

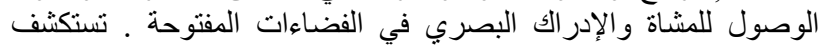

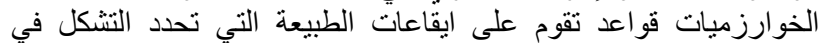

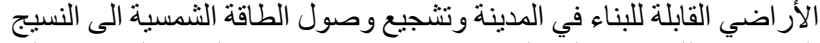

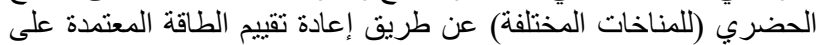

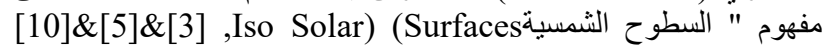

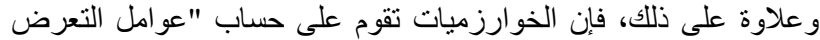
للسماء" و لأجز اءو واسعة من النسيج الحضري.

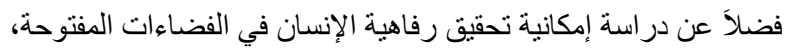

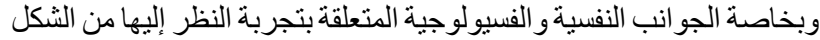

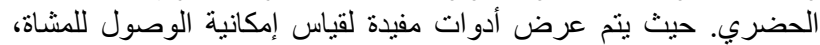

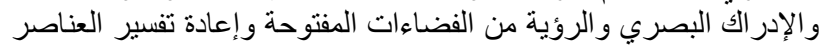

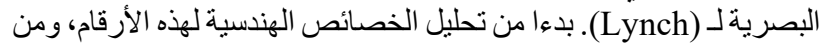

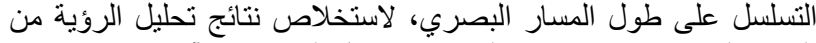

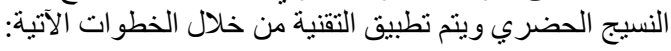

$$
\text { نموذج البناء الرقمي الحضري: }
$$

إن بناء نموذج البناء الرقمي الحضري يكون من خلال تصدير نموذج ثلاثي

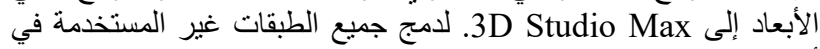

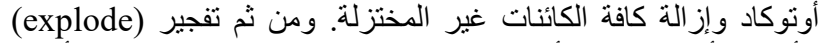

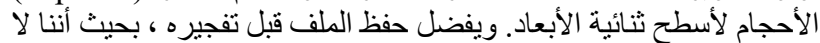

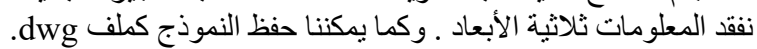

\section{بناء نموذج الارتفاع الرقمي في برنامج 3D Studio Max:}

يستورد هذا النموذج من برنامج 3D Studio Max. و عندما نستورد هذا

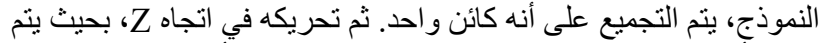

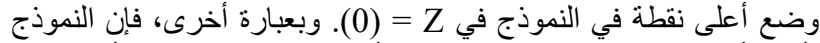

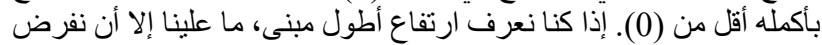

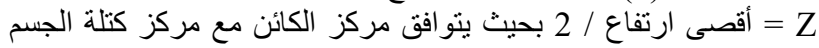
نفسه , كما في الثكل رقم 1.

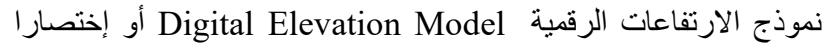

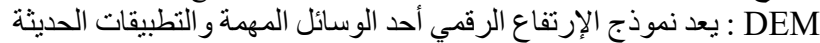

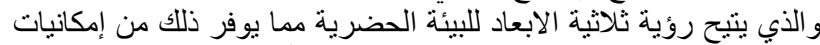

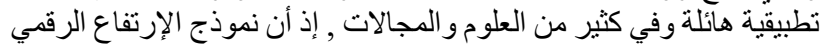

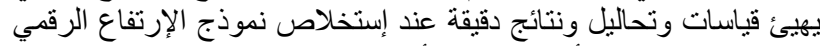

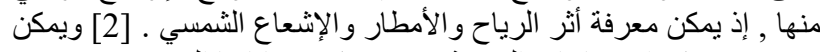

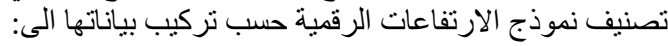

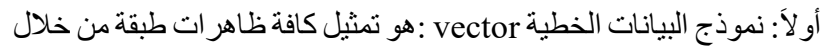

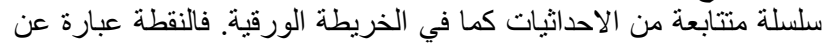

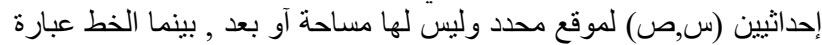

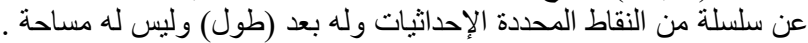

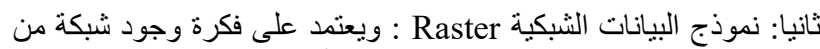

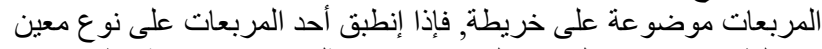

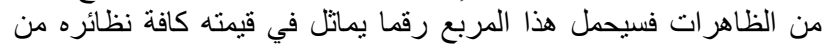

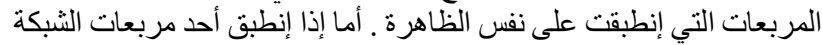

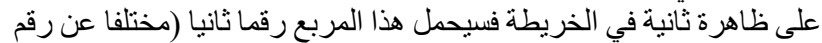

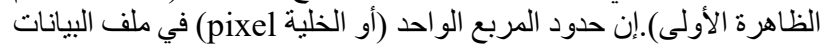

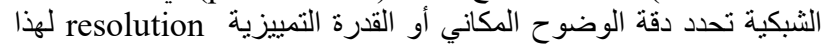

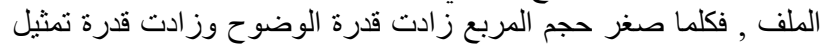

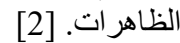

$$
\text { 2 } \quad \text { : } 2.1
$$

تعمل هذه التقنيات على محاكاة مخططات تصميمية بديلة على مستوى

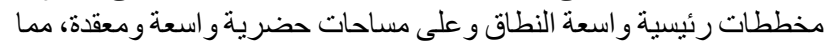

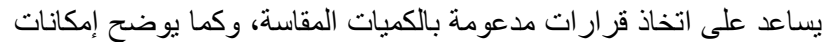

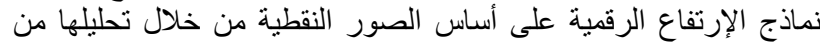

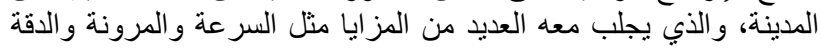

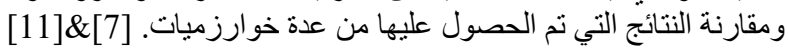

$$
\begin{aligned}
& \text { من أجل إنجاز هذه العملية نحتاج إلى نتبع الخطوات الآتية: } \\
& \text { تنبيت برنامج Matlab على جهاز الكمبيوتر . } \\
& \text { تثبيت برنامج 3D Studio Max على جهاز الكمبيوتر. }
\end{aligned}
$$

سلسلة من البرامج النصية (Matlab Codes). 


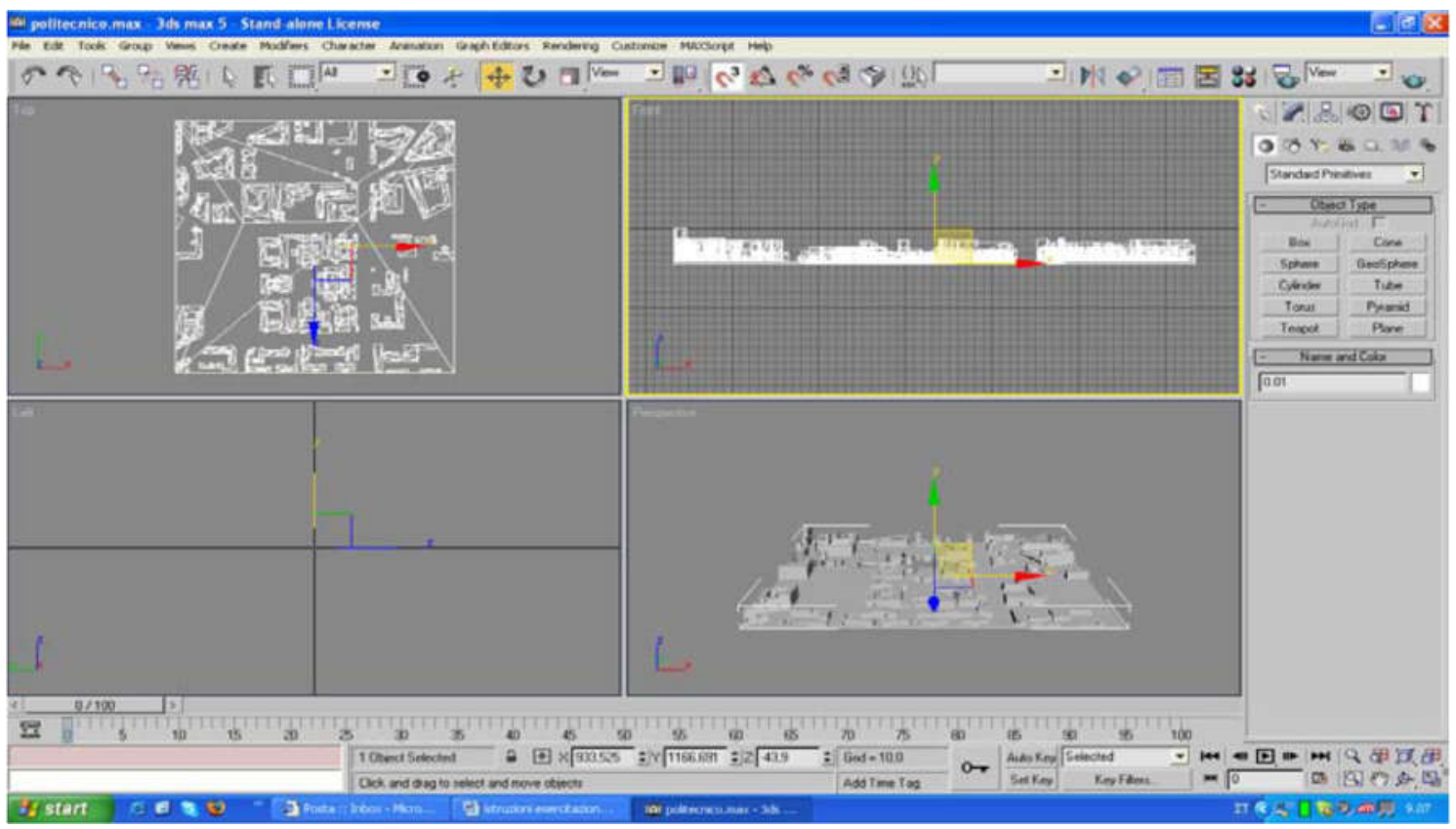

شكل 1: استير اد نموذج ثلاثي الأبعاد من Autocad في 3D Studio Max

[9]

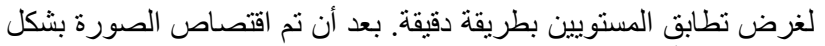

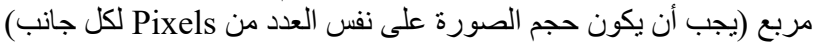
وحفظه S BMP أو B bits grey scale) TIFF).

\section{: Matlab Mتخدام رموز}

\subsection{3}

بعد أن تم إنشاء صورة نموذج الإرتفاع الرقمي (DEM) يمكننا استخدامها

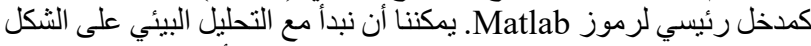

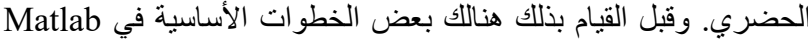
تهذف إلى التعريف في هذه التقنية:

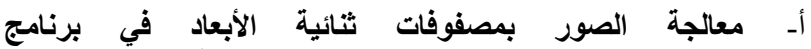

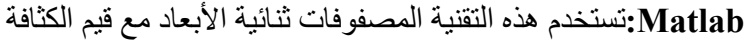

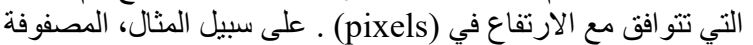
التالية اسمها العينة (مصفوفة 3×3 3) ركما في الثكل رقم 2 :
بعدها يصبح النموذج جاهز للإظهار (Render) والعمل العام على خلق نماذج

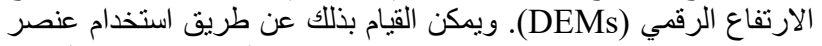

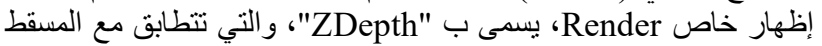
العلوي من بعد لا نهائي، حيث اللون الرمادي بكي بكسل (grey of the pixels)

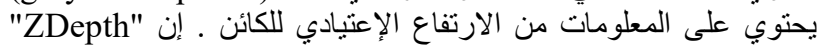

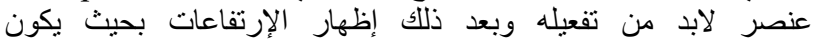

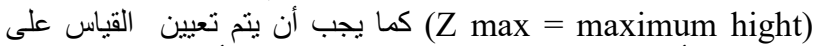

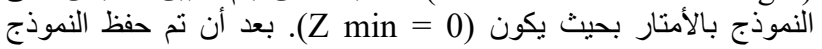

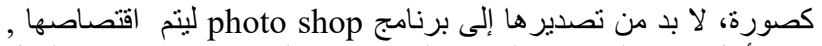

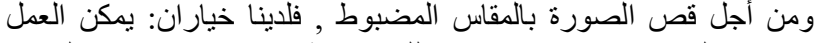

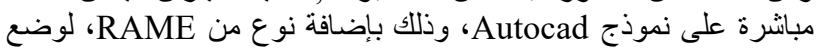

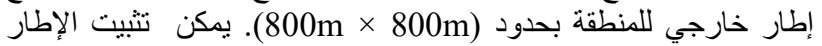
الخارجي في برنامج photo shop كطبقة منفصلة (على سبيل المثال

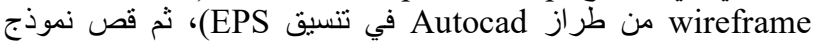

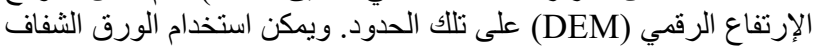
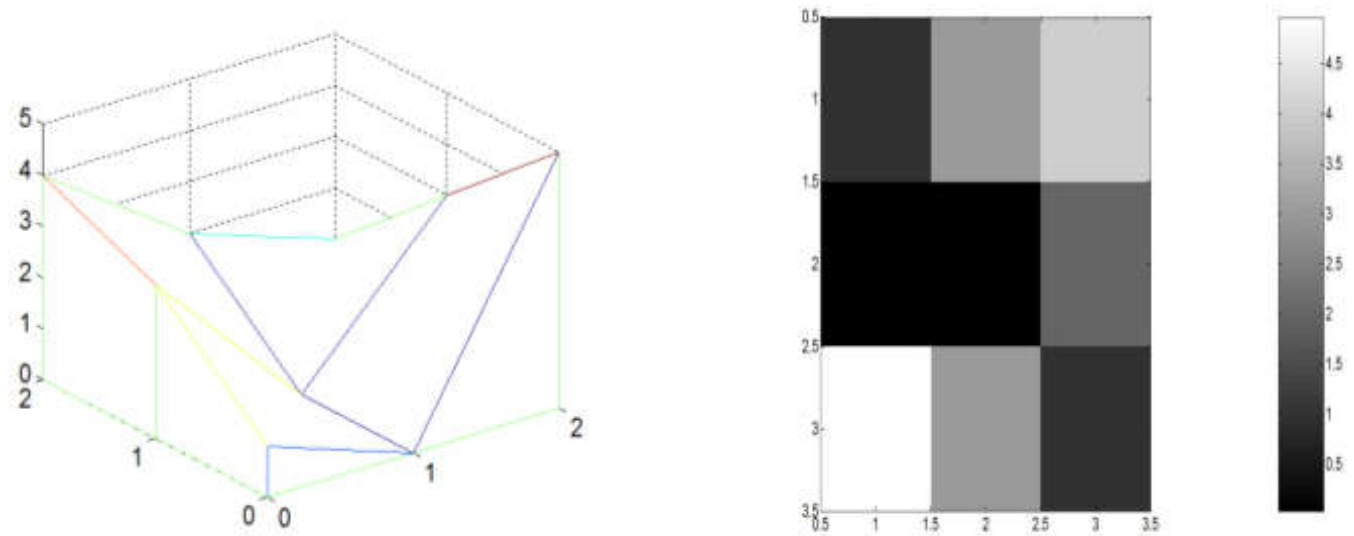

شكل 2: تخيل العينة مجمو عة ثنائية وثثلاثية الأبعاد . عينة = [4،3، 1، 2،0،0، 1،3، 
title ('shadow"s map');

colourbar;

colourmap(1-grey);

axis square;

إذا كانت الصورة تنطلب representation ثلاثي الأبعاد ، يمكننا أن نشير

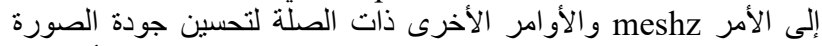

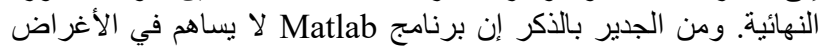
الكر افيكية بشكل مناسب.

$\operatorname{mesh}\left(b^{\prime}\right)$;

daspect $([1,1,1])$;

colourmap(1-grey);

view $(135,30)$

shading interp

camlight right

د- استخدام البرامج النصية: يتم استخدام نافذة (editor window) في

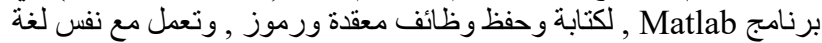

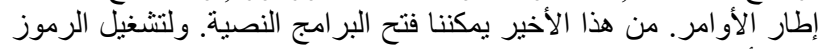

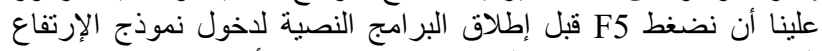
الرقمي (DEM) ومعايرة الصورة كما هو موضح الطنح أعلاه .

هـ - تصدير المخرجات (خرائط وأرقام): مخرجات النموذج هي الخرائط

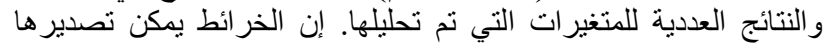

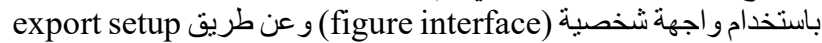

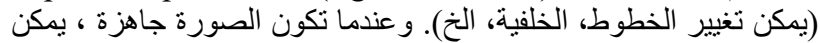

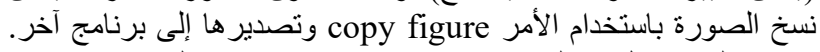
لإنشاء ملف مستقل مع الصورة مباشرة من Matlab ، بمكنك ترقيم (digit) الأمر imwrite

\section{( $A$, namefile, format, resolution)}

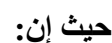

A: اسم مجمو عة / خريطة

Namefile: الاسم المعين إلى الصورة

format: شكل صورة

Resolution: (pixel) دقة الصورة بوحدة

على سبيل المثال، إذا كنا نريد لحفظ الصورة مع قناع للأسطح المبنية (mask of the built surfaces)

imwrite (built_mask, 'builtmask.tif', 'tif', 'Resolution', 300)

يمكن تعيين عدة خيار ات ثم الرجوع الى برنامج Matlab و الإستعانة بـ help و و (digit help imwrite on the command window) window

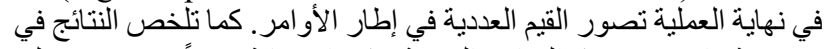

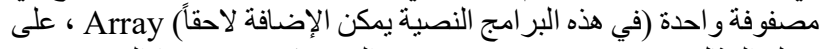

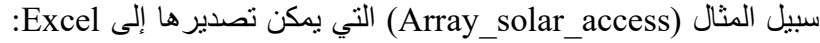
في الواقع، عن طريق آلنقر على اختيار Array on work space هني التى
ب - استير اد صورة على Matlab: عندما نستورد النموذج في بيئة

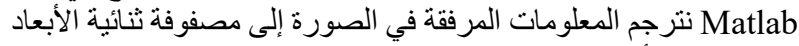

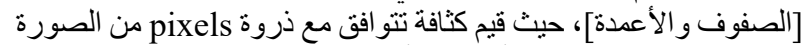

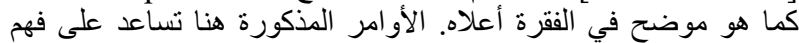

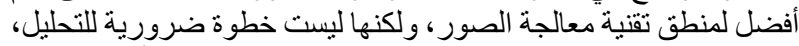

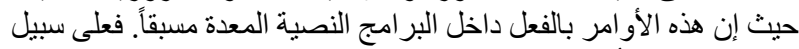

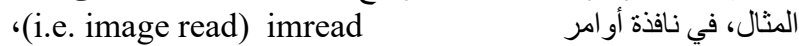

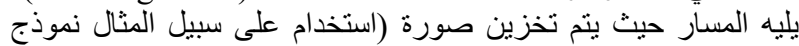

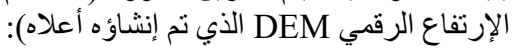

[a,c] = imread('C:|Users|...|models|DEM.tif');

$$
\text { a: يمثل اسم نؤشره على مصفوفة مع الصورة }
$$

ومن المهم لمعايرة صورة تعيين أبعادها المناسبة (الطول و العرض). و هذا

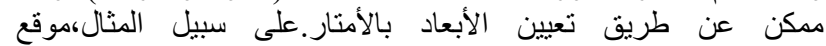
بقياس(800m × 800m).

widthx $=800$, widthy $=800$

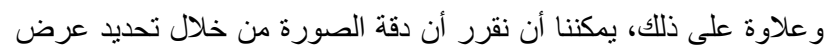

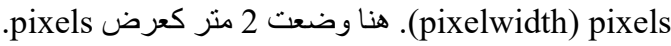

pixelwidth $=2$

$$
\text { يتم الحصول على حجم الصورة مع الصيغة: }
$$

sizex=widthx/pixe/width; sizey=widthy/pixe/width;

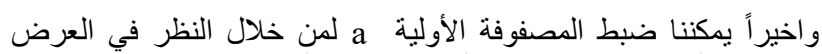

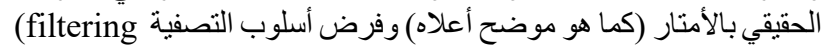

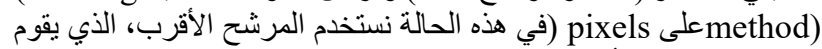

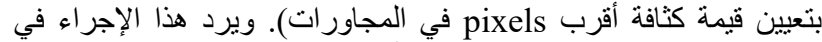
ويمكن أثنقاق معادلة المصفوفة (image re-size) imresize

b:

\section{b=imresize (a, [sizex sizey],'nearest');}

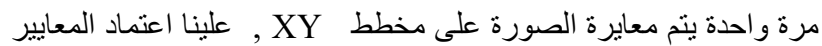

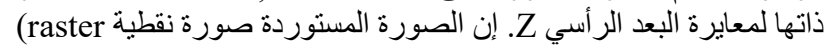
(image)

و هذا يعني أن قيم الكثافة الممكنة تكون (256) كقيم ممكنة، فمن الضروري

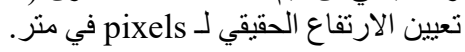

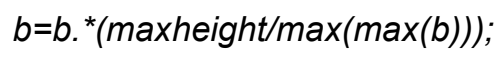

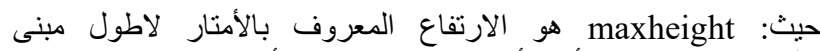

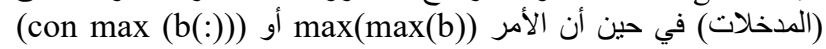
بحساب القيمة القصوى للمصفو القة 256 لونا، وفي هذه الحالة 255.

إذا كان لنا أن نلخص المدخلات اللازمة لمعايرة نموذج الإرتفاع الرقمي هي: (DEM)

maxheight, widthx, widthy, pixelwidth.

ج- تصور النتائج: من أجل رؤية مصفوفات(صور وخر ائط) يمكننا استخدام

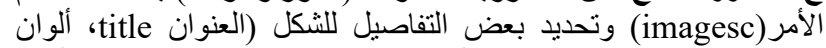
الخريطةcolourmap، حجم الألوان Scale of the colours، أبعاد (أبران الصورة (proportions of the image (الخريطةi ،

imagesc(f_total); 


$$
\begin{aligned}
& \text { متغير على مساحة العمل ، ومن هذه القيم يمكن النسخ و اللصق في برنامج حيث إن : }
\end{aligned}
$$

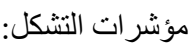

\section{morphology glasgow.m}

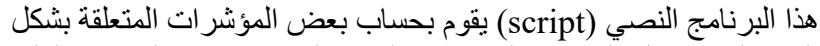

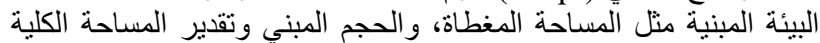

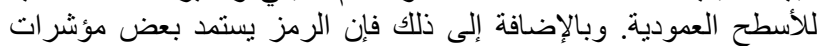
الكثافة, كما في الثكل رقبة 3.

ويكون من خلال البر امج النصية الثلاثة الآتية :

- morphology_glasgow.m

- $\quad$ shadowsonadem_glasgow.m

- $\quad$ skyviewfactor_glasgow.m
QUANTITA' DENSITA'

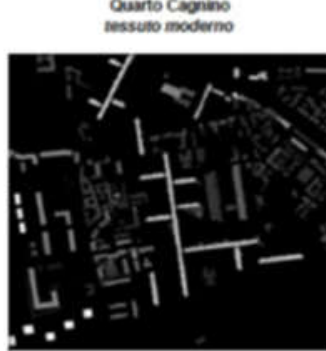

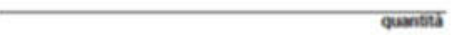
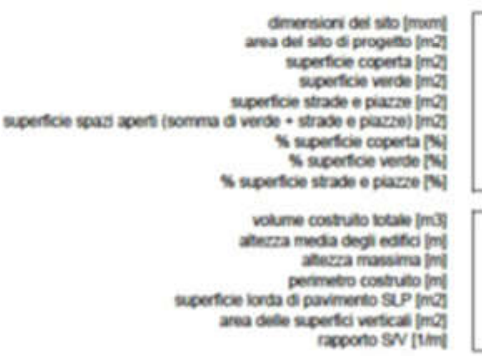

madica demina

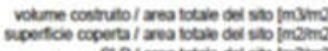

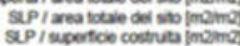
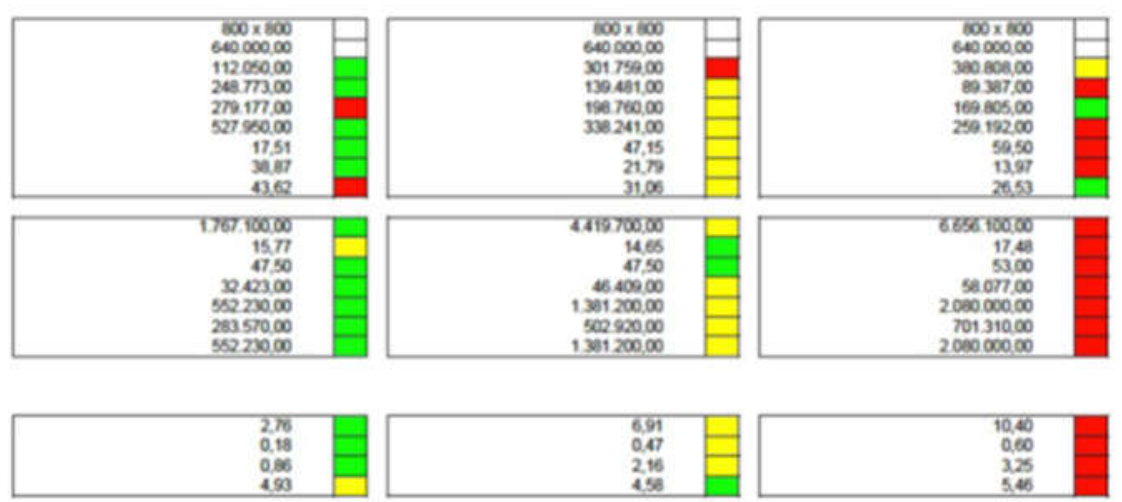

شكل 3: مؤشرات التشكل morphology indicatprs/ كثافة البناء

[9]

لكل القيم بالساعة على نفس الخريطة. كما يتم التعبير عن نتائج رقمية للامتار

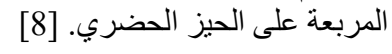

المدخلات اللازمة لتثغيل البرنامج النصي هي:

معايرة للصورة (أبعاد منطقة بالمتر وأقصى ارتفاع على الموقع).

ويوم من أيام السنة المختار.

خط عرض الموقع.

فثثلا: يوم = 21 / الثهر = 12 / خط العرض = latitude shadowsonadem glasgow.m: التظليل

إن هذا البرنامج النصي يحسب مسار الطاقة الثمسية وخر ائط التظليل

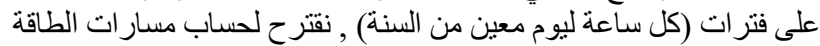

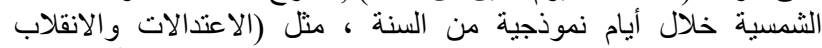

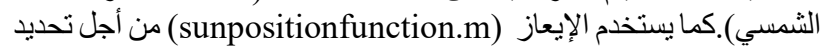

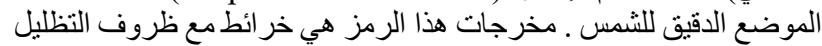

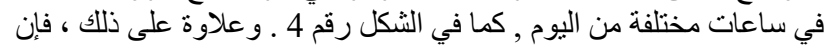
الخريطة الاصطناعية المسماة shadows density map ، تمثل القيم العليا 


\section{Tools/Urban Metabolism/SunScapes/Shadow Casting}

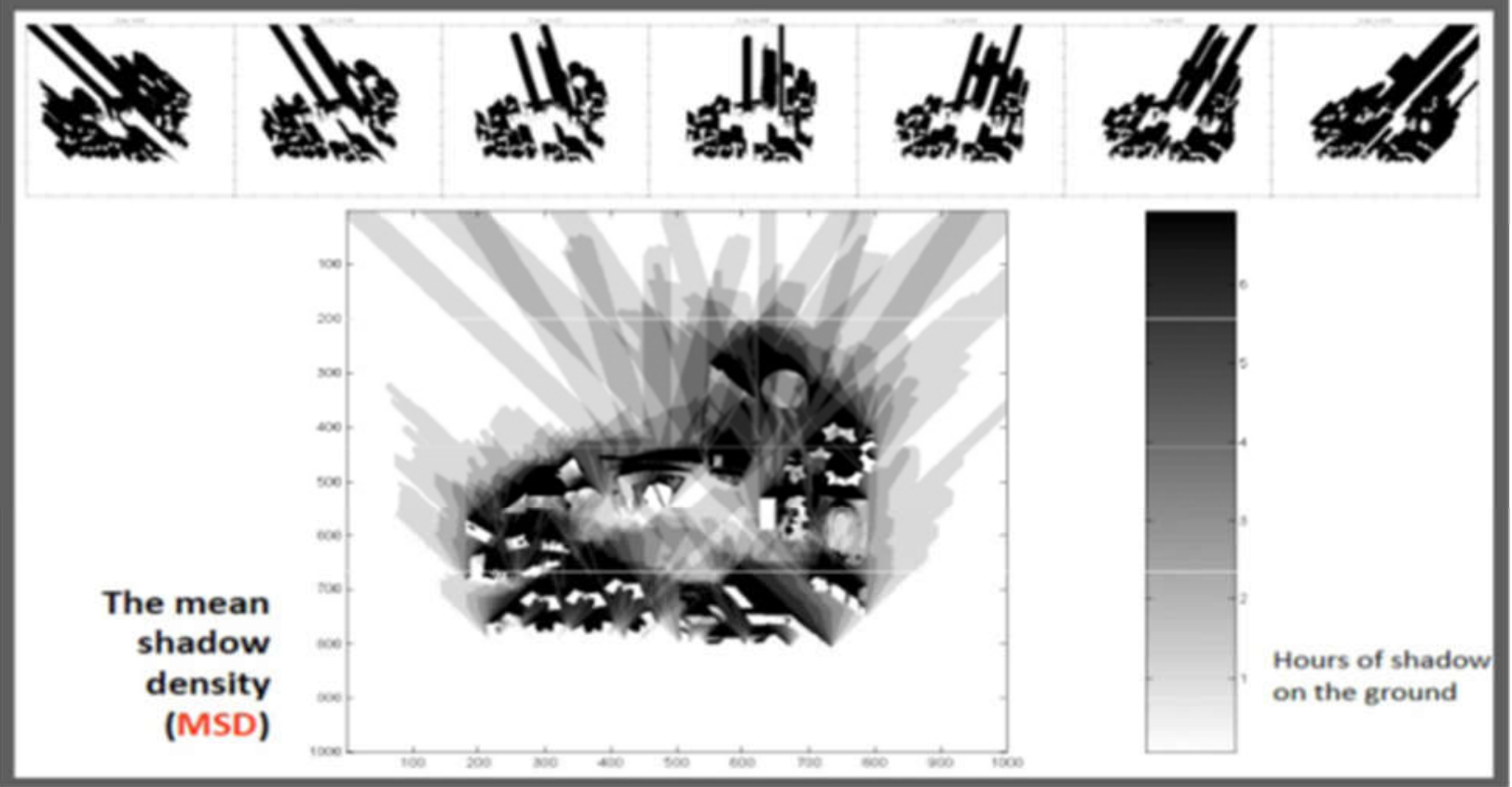

شكل 4: مؤشر ات التشكل morphology indicatprs/خرائط الظل

[9]

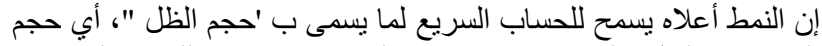

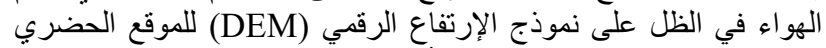

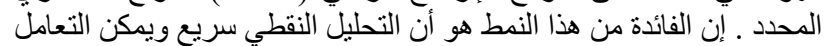

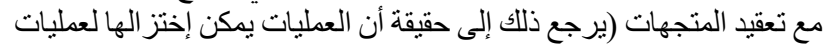

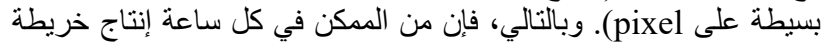

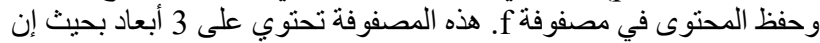
قيم X و قيم Y (0 أو 1 1) من خريطة الظل و الساعة.

\section{$f \_$tot(:,:,hour $)=f$}

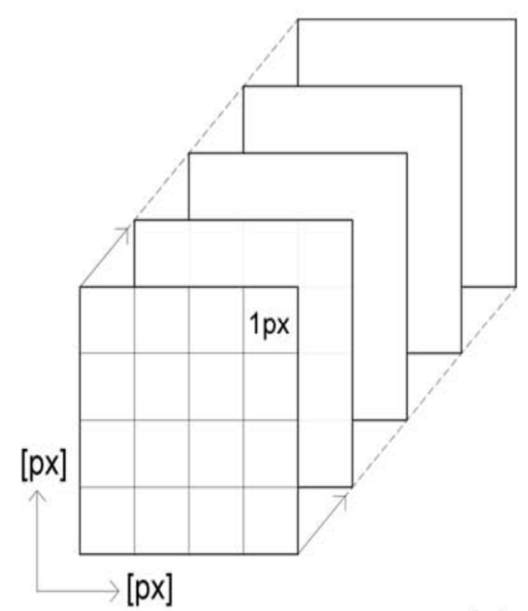

(a)

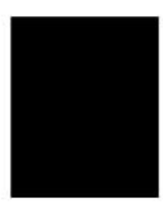

(b)
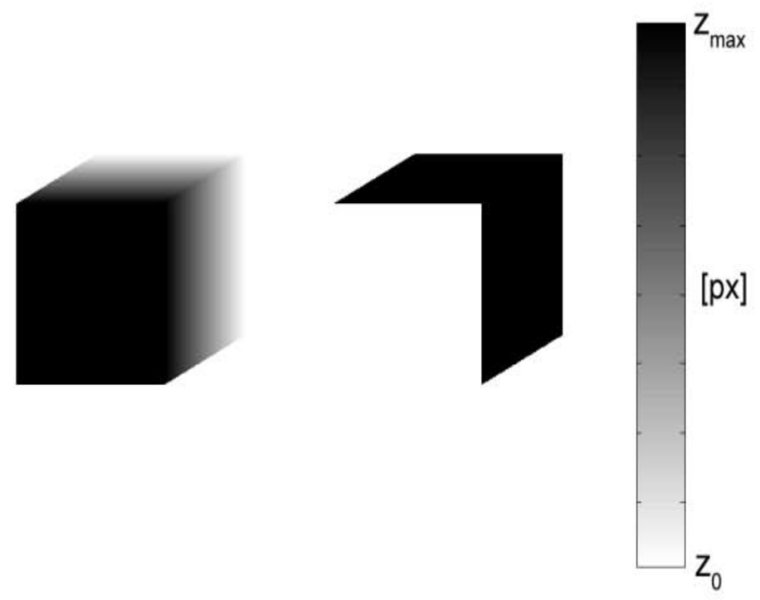

(c)

(d)
يشتق النمط الفر عي أدناه من البرنامج النصي لسقوط الظل, ويمثل أساسا

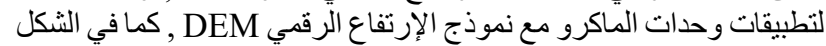

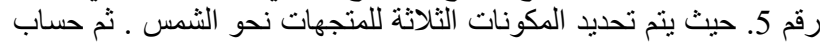

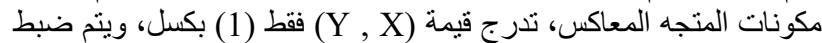

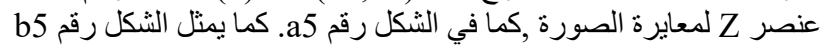

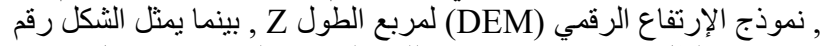

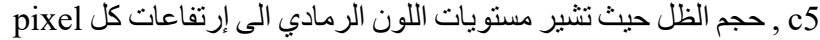

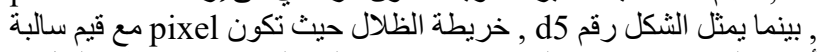
أُو قيم الصفر في منطقة الضوء. بينما تكون القيم الموجبة في منطقة الظل.

شكل 5: حساب حجم الظل من خلال معالجة الصور من DEMs (a) : ترجمة الماكرو ، DEM (b) لمربع الطول Z) (c) (c) حجم ظله حيث تثير مستويات الرمادي الى إرتفاعات كل تر ل

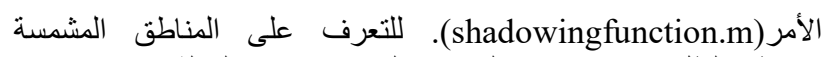

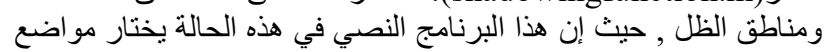
عشو ائية على قبة السماء , [12] كما في الثكل رقم 6. skyviewfactor_glasgow.m : معامل التعرض للسماء إن هذا البرنامج النصي يحسب معامل التعرض للسماء (SVF) من النسيج

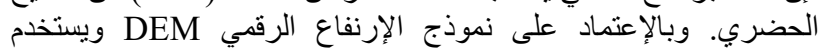


SKY - VIEW FACTORS

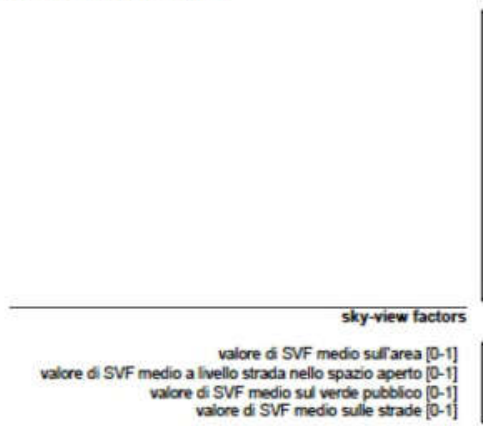
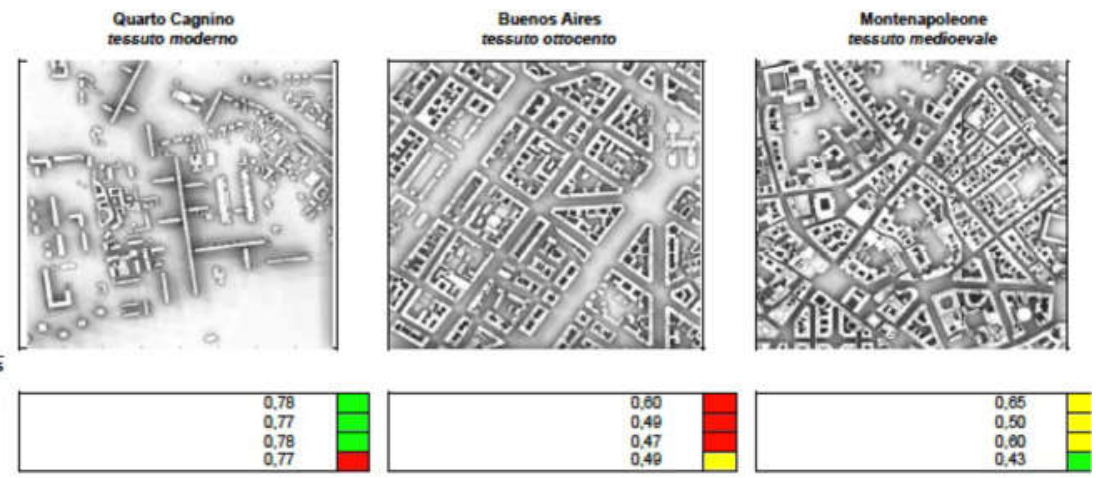

Oggetto del calcolo

Calcolo del fattore di vista del cielo. Rappresenta rapertura di tutti i punti alla volta celeste. La porzione di cielo visibile da un punto dello spazio è direttamente collegabile alla capacità d suolo di riflettere verso il cielo il calore accumulato durante la giomata, le mappe ricavate rappresentano quindi un modello semplificato per descrivere risola di calore urbana notturna durante il periodo estivo.

Risultati

I valori più alti, quelli che indicano una ampia veduta del cielo, si registrano sia per quanto riguarda gli spazi aperti, che il verde pubblico, che le strade, nel tessuto moderno.

I tessuti medioevale e ottocentesco della citta presentano spazi con minor visibilità della volta celeste. Ma questi sono costituiti per la maggior parte dalle cort interne, (che per la loro ger metria di spazi hanno potenzialmente una capacità di raffrescamento notturna costituita dal raffreddamento delle coperture per irradiazione e al deflusso per convenzione verso il basso dell'aria fredda con conseguente raffrescamento della corte), mentre le strade e le piazze, soprattutto nel caso di Buenos Aires presentano una discreta visibilita.

HW RATIO
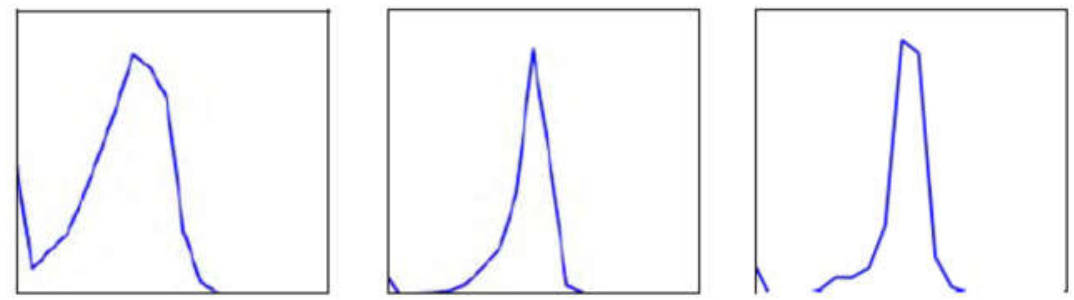

شكل 6: مؤشر ات التشكل morphology indicatprs / خرائط الظل

[9]

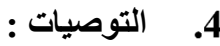

إعادة النظر في المعايير التخطيطية القائمة وفقاً للإستنتاجات التي توصل إليها

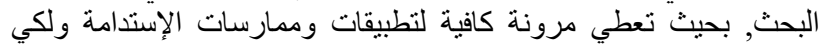
تسيطر وتقلل من الممارسات الخاطئة في التخطيط و التصميم و التنفيذ بحيث التيث تتكامل تلك المعايير مع المعايير العالمية والمحلية.

ضرورة البحث في المؤشر ات البيئية في العمارة التي تقوم بحساب أداء الطاقة

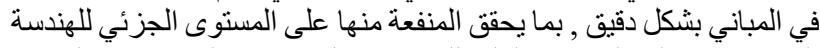

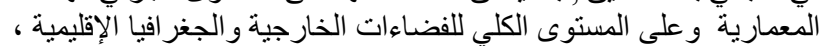

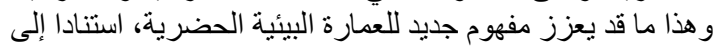

استر اتيجيات تصميم جديدة وقو اعد تنبؤية لتوليد الثكل , مشتقة من المؤشرات

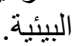

الإستفادة من النتائج الأولية التي توصل إليها البحث في تطوير السياق

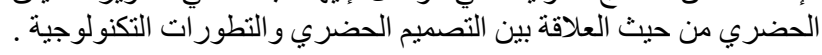

ضرورة مواكبة التطور الرقمي نظراً للأهمية المتزايدة للمعلومة ثلاثية

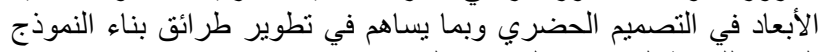
الرقمي للوسط العمر اني بالكقاييس الكبيرة .
: الإستتنتاجات

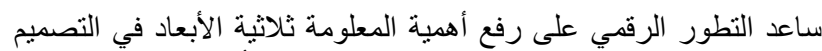

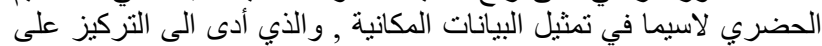

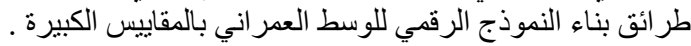

إن تحقيق الجودة البيئية في المساحات الحضرية. يعتمد على العلاقة القائمة

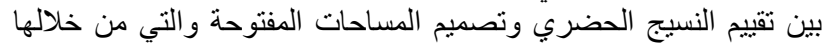
تتحدد نوعية البيئة الحضرية ومدى نجاح المين المدينة.

أضحت مجالات استخدام نماذج الارتفاعات الرقمية وتطبيقاتها الواسعة

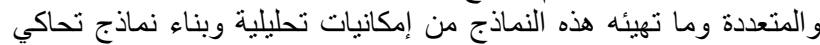

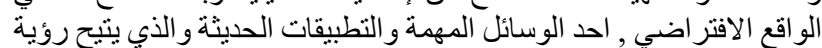

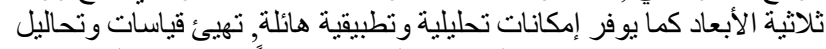

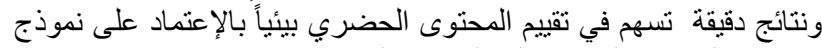

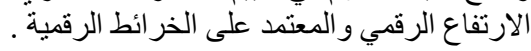

إن تقييم المحتوى الحضري بيئيا بالإعتماد على الوسط الرقمي يفتح آفاقاً من

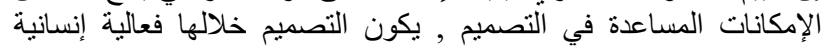

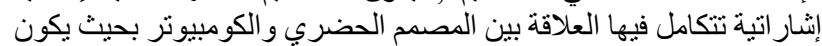

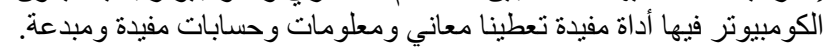


[6] Moughtin, C. (1999), "Urban Design - Methods and Techniques", Butterworth - Heinemann Ltd., Britain

[7] Morello, E., Ratti, C. 2009. "A Digital Image of the City: 3-D isovists in Lynch's Urban Analysis". Environment and Planning B: Planning and Design , 36, 837-853

[8] Ratti C., Richens P., 2004, "Raster Analysis of Urban Form", Environment and Planning B: Planning and Design, 31:2, 297-309

[9] Sergio Porta, Ombretta Romice, and Tutors , 2009/10 ,ANALYSIS BRIEF Comparative analysis of urban fabrics, 16-22.

[10]Zlatanova and stoter2003, Stoter,J.E. and S. Zlatanova ,2003, "Visualising and Editing of 3D Objects Organized in a DBMS", EUROSDR workshop, Rendering and Visualization , Enschede, The Netherlands

\section{Internet}

[11] Riterion Planners, 2014, $17-$ 5,2015,http://www.crit.com/documents/planuser guide

[12]U.S. Green Building Council ,2014, 30-4-2015, http://www.usgbc.org/ShowFile.aspx?Document $\mathrm{ID}=6146$

[13] Hofstra University, 2015, 13-2-2015, http://people.hofstra.edu/geotrans/eng/ch2en/me nth2en/ch2m1en.html

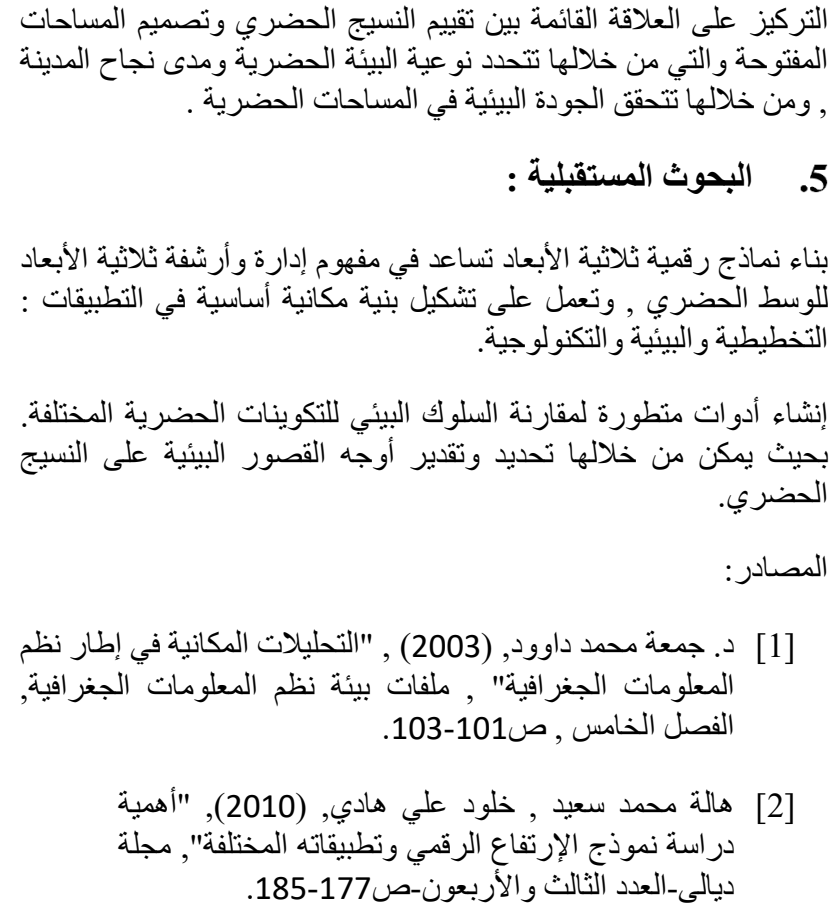

\section{Refrences}

[3] Criterion Planners Engineers, 2001, INDEX Plan Builder User Guide, Portland

[4] Gosling, D. (1984), "Definitions of Urban Design", AD publications Ltd, Great Britain

[5] Kanski, K.J., (1963), Structure of Transportation Networks: "Relationships Between Network Geometry and Regional Characteristics", University of Chicago Press, Chicago 


\title{
Evaluation of Environmentally Urban Content Depending on Digital Medium
}

\author{
Mazin Ismael Raheem ${ }^{1, *}$
}

${ }^{1}$ Department of Architectural Engineering, University of Baghdad, Baghdad, Iraq, archi.mazin@yahoo.com

* Corresponding author: Mazin Ismael Raheem and email: archi.mazin@yahoo.com

Published online: 31 August 2019

Abstract- This research suggest a technique for evaluating the urban content environmentally depending on digital media, throughout identifying the creative analytic techniques that develops the simple urban raster which is known as Digital Elevation Models( DEMs)Additionally, we prepare a group of systems that adopt 3D representation through simple drawing instruments, display environment and 3D spacial analysis. They are supporting programs for the concept of 3D representation and analysis, not the building specially in large scales. Now the research problem emerged, represented by: obviously deficient acknowledgment, in practical and academic media, in using digital medium that help to predict and evaluate, environmentally; the urban content. So, this research aims at: delineating some urban form linked environmental pointers through simple aids use (groups of scripts) that predicts the environmentally evaluated, the urban content: through designing process. This research depends in its academic and practical frames on the concept that it acts by developing effective strategic materials for analyzing and delineation the urban form and measuring the environmental pointers reaching to conclusion and recommendations. 